\title{
International Trends Analysis for Subject of Hydrogen Production with Water Electrolysis
}

\author{
Fei Pengfei ${ }^{1}$, Zhang Chao ${ }^{1,2 *}$ \\ ${ }^{1}$ National Science Library, Chinese Academy of Sciences, Beijing, China \\ ${ }^{2}$ School of Economics and Management, University of Chinese Academy of Sciences Beijing, China
}

\begin{abstract}
Since the $21^{\text {st }}$ century, the domestic and foreign energy structure has been deeply adjusted and therefore the study about hydrogen production with water electrolysis becomes a hotspot. This paper makes bibliometric analysis for related indexes in the field of hydrogen production with water electrolysis, for example, output scale of articles, academic influence, discipline layout, and international cooperation, and compares China's strength with other countries around the world to obtain development trend of the technology in the world, thus formulating related development strategy and discipline policy for the technology and providing useful reference for corporate investment decision-making and researchers in selecting research direction.
\end{abstract}

\section{Introduction}

The hydrogen is widely used in the chemical, energy and material industry as the chemical material. In the context of domestic and foreign adjustment in energy structure in the past decades of years, the concept "hydrogen economy" has been improved and the industry chain of hydrogen energy has also been developed gradually. Featured with carbon-free environmental protection and flexibility, the hydrogen production technology with water electrolysis has attracted a lot of attentions ${ }^{1-4}$. In order to accurately obtain development status and potentials and draw a "topographical map" for the development trends, this paper focuses on the hydrogen production with water electrolysis and analyzes international trends and subjects of related studies with literature database and a progressive plane-to-point framework based on the qualitative investigation and expert consultation. The purpose is to analyze domestic and foreign research status and technical hotspot of the field and further demonstrate China's research status in the field of hydrogen production with water electrolysis.

\section{Data source and analysis methods}

In order to retrieve articles and reviews related to the hydrogen production with water electrolysis, a retrieval strategy ${ }^{1}$ is created based on terms provided by the experts. A total of 37726 articles were filtered by literature types ("Article" and "Review") in June 2020. Then the data was analyzed with Excel and Derwent Data Analyzer (DDA).

\section{Results}

\subsection{Analysis for output scale of related studies}

\subsubsection{Trend analysis}

From the view of distribution of SCI articles related to the hydrogen production with water electrolysis by time, as shown in Figure 1, the hydrogen production with water electrolysis was studied initially from 1990s internationally and few studies were developed before 1990. For example, the "water electrolysis and oxygenhydrogen chain" related achievements were published on Helvetica Chimica Acta in 1921. The annual average number of articles of the field exceeded 1000 after 2013 and increased with growth rate $25 \%$. As a result, the global studies related to the hydrogen production with water electrolysis develop fast and reach a certain scale.

\footnotetext{
${ }^{1}$ Retrieval strategy: Subject: ("water electroly*" OR "water split*" OR "oxygen evolution reaction" OR "hydrogen evolution reaction") Index $=S C I-E X P A N D E D$ Time span $=$ All years
}

* Corresponding authors. E-mail addresses: zhangch@mail.las.ac.cn 


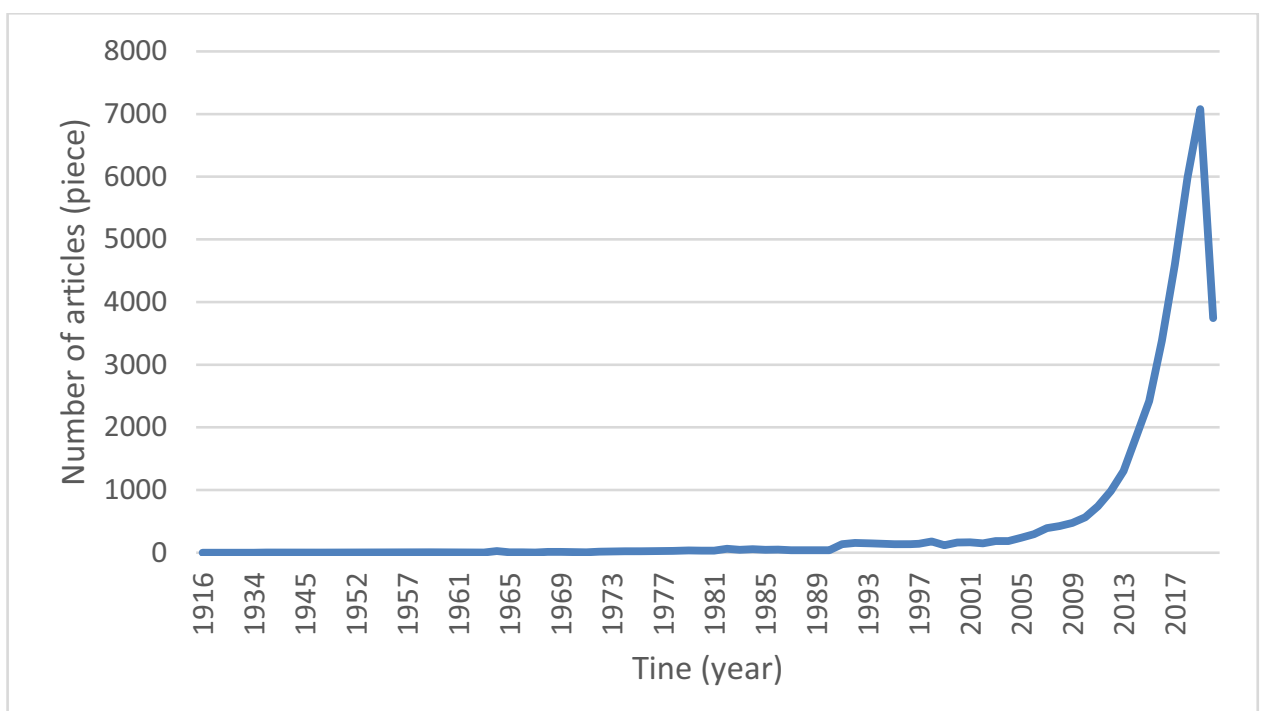

Figure 1 Distribution of SCI articles related to the hydrogen production with water electrolysis by time

\subsubsection{National / regional distribution}

Over 130 countries and regions around the world have developed studies related to the hydrogen production with water electrolysis. Figure 2 shows the top 20 countries. The top 10 countries include China, USA, Korea, Japan, Germany, India, Australian, UK, Singapore, and Canada, with number of articles over $70 \%$ of the total articles related to the hydrogen production with water electrolysis.

From the view of country, China is dominant in the study of this field, of which the number of articles is $34.25 \%$ of the total. The number of articles is over 10000 for China and about 6000 for USA; about 2000 for Korea, Japan and Germany; about 1000 for India, Australia, UK, and Singapore. Therefore, there is a large difference between China and other countries.

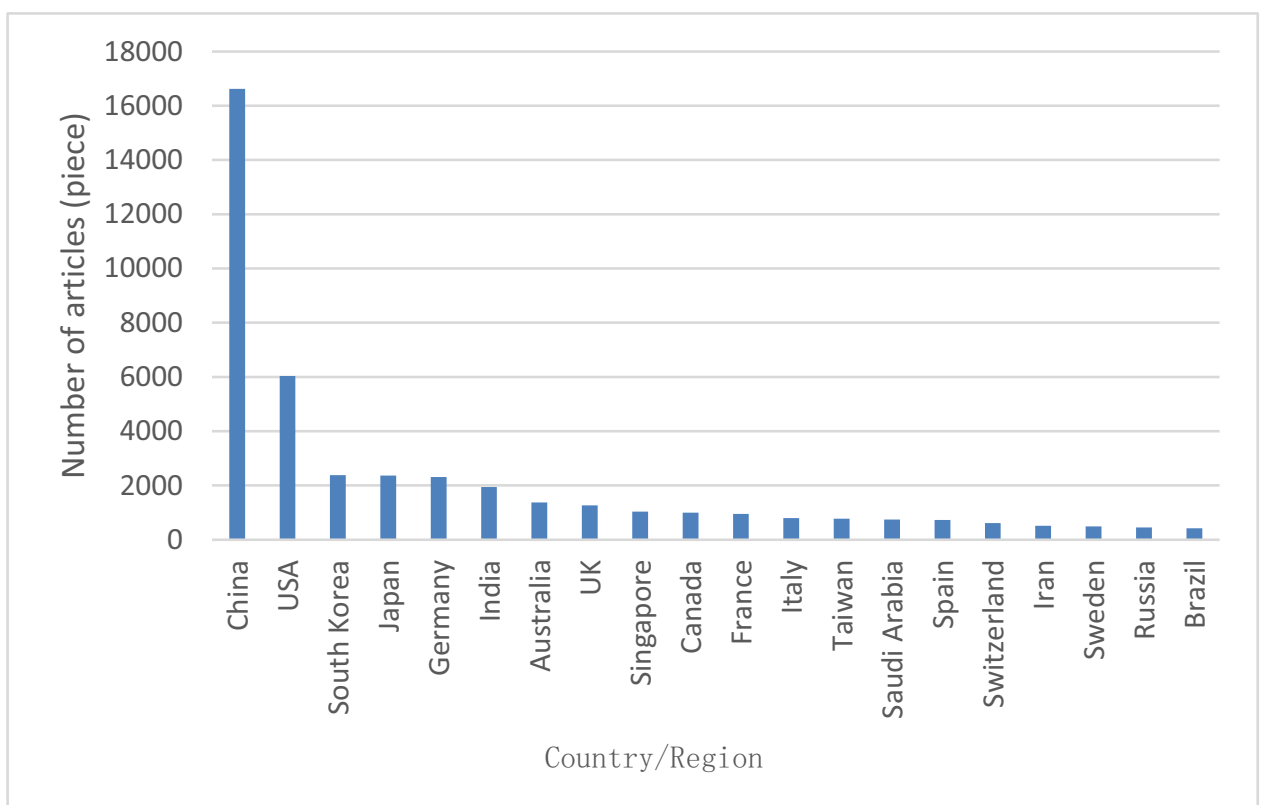

Figure 2 Distribution of SCI articles related to the hydrogen production with water electrolysis by county/region

\subsubsection{Institutional distribution}

\subsubsection{Distributions of global institutions}

The number of articles related to the hydrogen production with water electrolysis is over 400 for the top 10 institutions around the world, of which 6 institutions have published over 500 related articles, as shown in Table 1. Among the top 10 institutions, except the No. 4 institution from Singapore and No. 9 institution from Japan, all the rest institutions come from China. The top 5 institutions include Chinese Acad Sci, Univ Chinese Acad Sci, Univ Sci \& Technol China, Nanyang Technol Univ, and Tianjin Univ, where the number of articles is 2592 for Chinese Acad Sci being the first level of the field; the number of articles is over 500 for Univ Chinese Acad Sci, Univ Sci \& Technol China, Nanyang Technol Univ, Tianjin Univ and Tsinghua Univ being the second level of the field; the number of articles is over 400 for Soochow Univ, Beijing Univ Chem Technol, Univ Tokyo, and Jilin Univ being the third level of the field. 
Table 1 Distribution of SCI articles related to the hydrogen production with water electrolysis by institution

\begin{tabular}{cccc}
\hline Ranking & Author's institution & $\begin{array}{c}\text { Number of } \\
\text { articles }\end{array}$ & $\begin{array}{c}\text { Country } \\
\text { (Region) }\end{array}$ \\
\hline $\mathbf{1}$ & Chinese Acad Sci & 2592 & China \\
\hline $\mathbf{2}$ & Univ Chinese Acad Sci & 801 & China \\
\hline $\mathbf{3}$ & Univ Sci \& Technol China & 755 & China \\
\hline $\mathbf{4}$ & Nanyang Technol Univ & 664 & Singapore \\
\hline $\mathbf{5}$ & Tianjin Univ & 601 & China \\
\hline $\mathbf{6}$ & Tsinghua Univ & 528 & China \\
\hline $\mathbf{7}$ & Soochow Univ & 479 & China \\
\hline $\mathbf{8}$ & Beijing Univ Chem Technol & 451 & China \\
\hline $\mathbf{9}$ & Univ Tokyo & 433 & Japan \\
\hline $\mathbf{1 0}$ & Jilin Univ & 422 & China \\
\hline
\end{tabular}

\subsubsection{Analysis of institutional cooperation}

It can be seen from the analysis for cooperation among the top 20 institutions by number of articles in the field that various institutions of each country have developed wide cooperation with domestic and international counterparts, as shown in Figure 3-5.

- For example, the representative Chinese Acad Sci has established close cooperation with Univ Chinese Acad Sci, Univ Sci \& Technol China, Tianjin Univ, Tsinghua Univ, Beijing Univ Chem Technol, Univ Tokyo, Harbin Institute of Technology, Nanjing University, Peking University, Sun Yat-sen University, Shandong University, Soochow Univ, and Central South University, where the number of published articles is largest for Univ Chinese Acad Sci and the number of co-published articles of both institutions is 559. Internationally, Chinese Acad Sci cooperates with foreign universities for research, including Nanyang Technol Univ, National University of Singapore, and University of Tokyo, where the number of copublished articles with Nanyang Technol Univ is 23 .

- The Nanyang Technol Univ ranks the No. 4 in the number of articles related to the hydrogen production with water electrolysis, and co-published 25 related articles with National University of Singapore. Besides, this institution has established close cooperation with Univ Chinese Acad Sci, Univ Sci \& Technol China, Tianjin Univ, Tsinghua Univ, Beijing Univ Chem Technol, Harbin Institute of Technology, Nanjing University, Peking University, Soochow Univ, and University of Tokyo respectively, where the number of co-published articles with Chinese Acad Sci, Tianjin Univ, and Nanyang Technol Univ is 10.

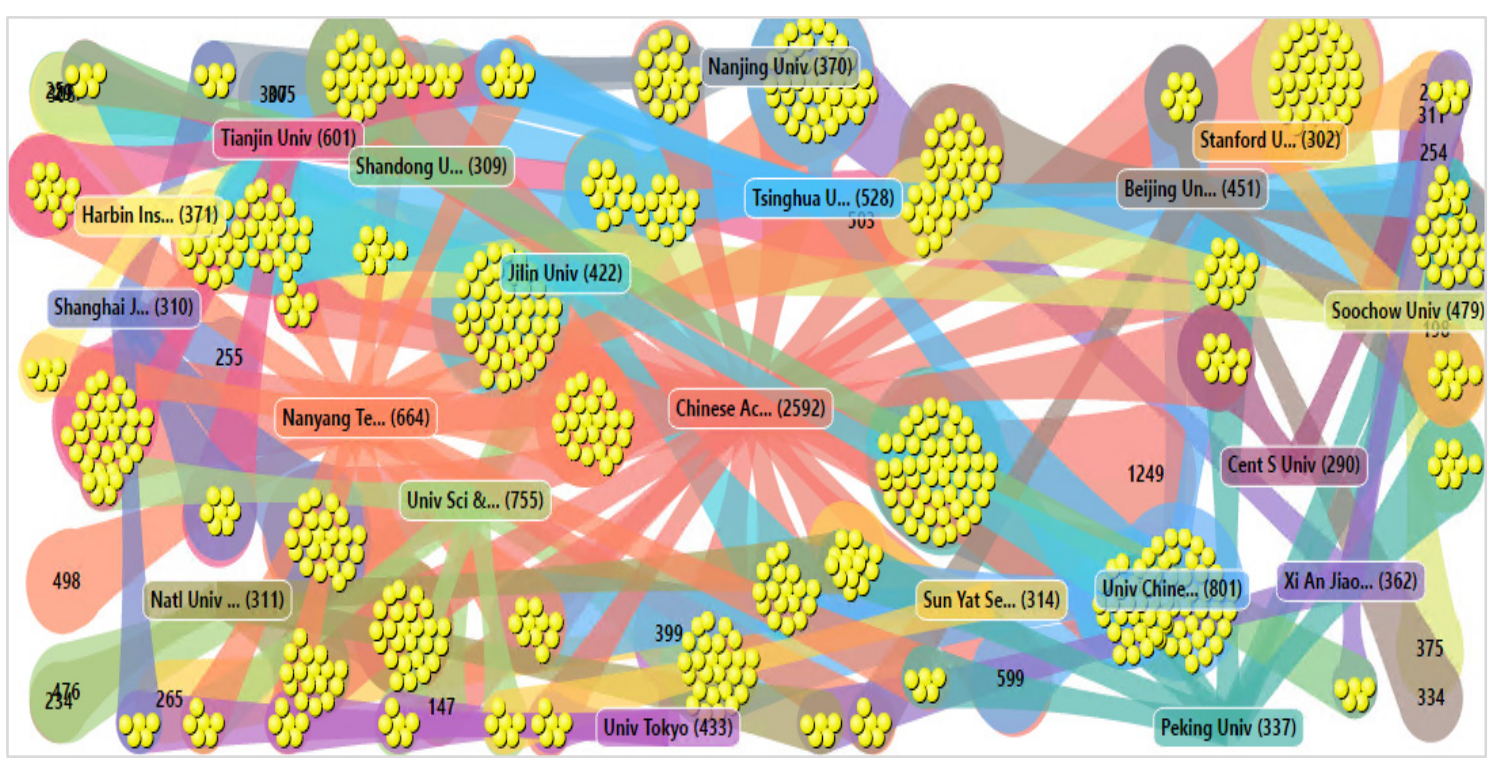

Figure 3 Distribution of institutions with cooperation in the study on hydrogen production with water electrolysis 


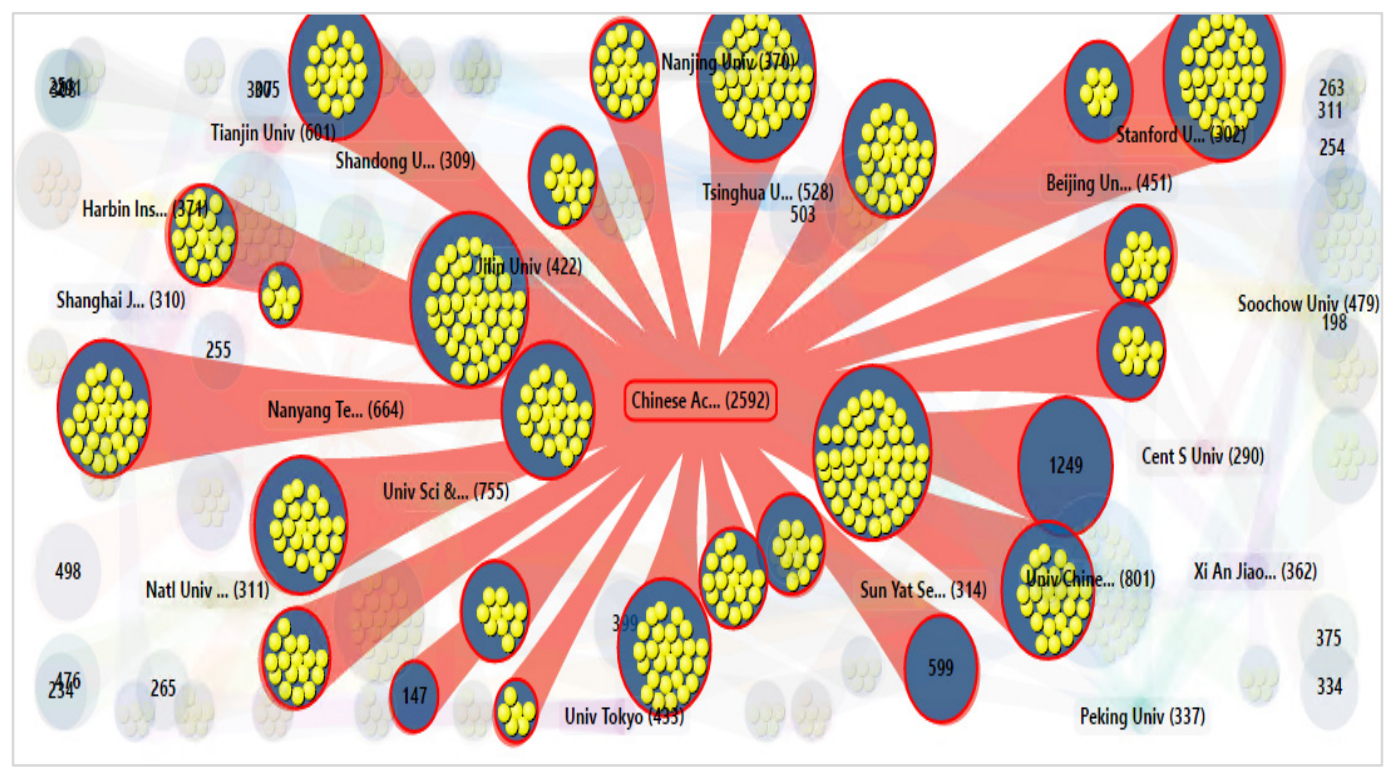

Figure 4 Cooperative institutions of Chinese Acad Sci

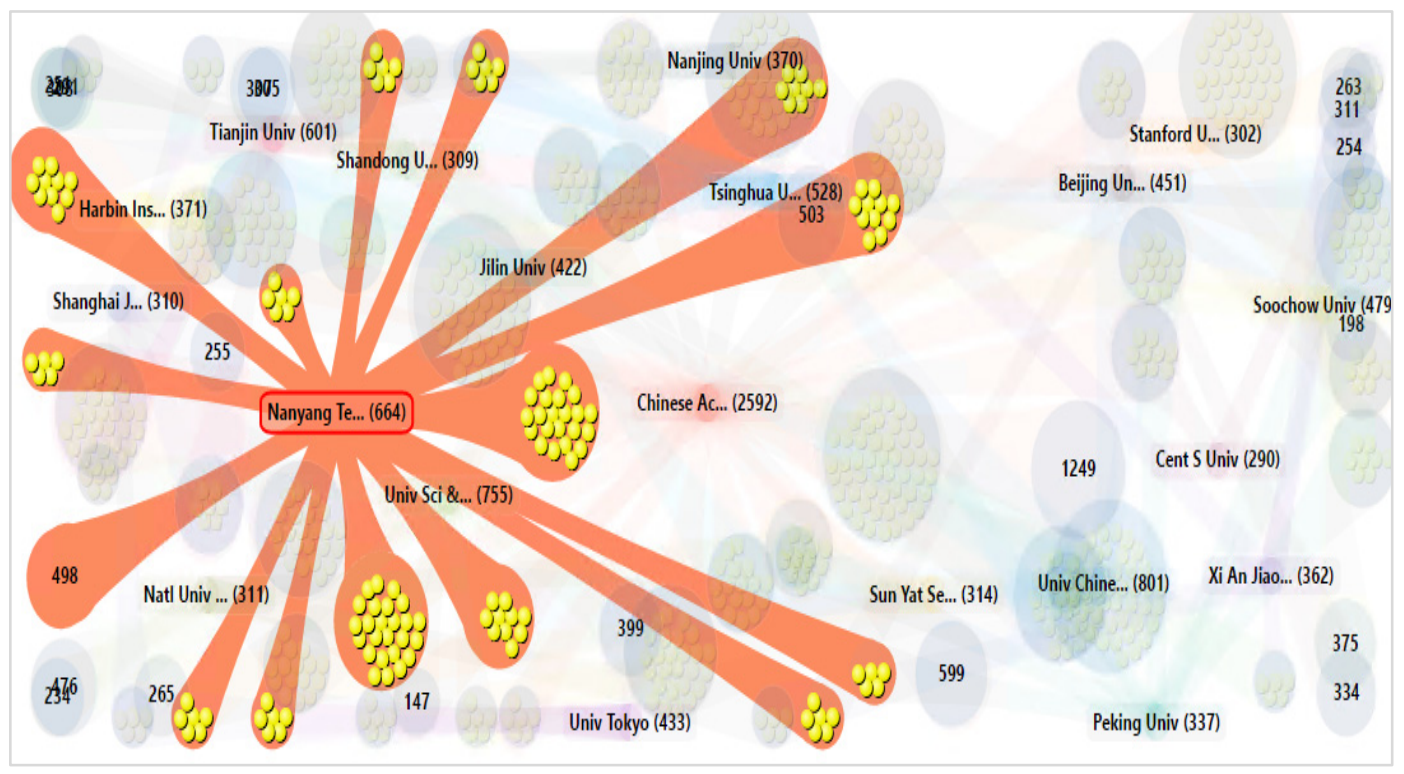

Figure 5 Cooperative institutions of Nanyang Technol Univ

\subsection{Analysis of citation frequency by country/region}

The citation frequency of articles related to the hydrogen production with water electrolysis around the world is analyzed and accumulated by country to calculate the average citation frequency, as shown in Table 2. The total citation frequency and average citation frequency indicate influence of such articles, where the total citation frequency indicates influence of a country in the field and the average citation frequency indicate the degree of attention to such articles.

From the view of total citation frequency, the top 10 countries include China, USA, Japan, Germany, Australia, Singapore, Korea, UK, Saudi Arabia, Switzerland and Canada. The total citation frequency is 533914 for China, which is larger than other countries. However, the average citation frequency is 32.12 for China, ranking in No. 14, but its influence of articles falls behind the USA, Spain, UK, Switzerland, Italy, Canada, and France. Although USA falls behind China in the number of articles, ranking in No. 2 , the average citation frequency is 64.53 , ranking in No.1.

Table 2 Citation frequency of SCI articles related to hydrogen production with water electrolysis by country/region

\begin{tabular}{lllllll}
\hline \multirow{2}{*}{ No. } & \multirow{2}{*}{ Country/Region } & \multirow{2}{*}{$\begin{array}{c}\text { Number of } \\
\text { articles }\end{array}$} & \multicolumn{2}{c}{ Total citation frequency } & \multicolumn{2}{c}{ Average citation frequency } \\
\cline { 4 - 7 } & & Frequency & Ranking & Frequency & Ranking \\
\hline 1. & China & 16621 & 533914 & 1 & 32.12 & 14 \\
\hline
\end{tabular}




\begin{tabular}{lllllll}
\hline \multirow{2}{*}{ No. } & \multirow{2}{*}{ Country/Region } & $\begin{array}{c}\text { Number of } \\
\text { articles }\end{array}$ & & \multicolumn{2}{c}{ Total citation frequency } & \multicolumn{2}{c}{ Average citation frequency } \\
\cline { 5 - 7 } & & Frequency & Ranking & Frequency & Ranking \\
\hline $\mathbf{2 .}$ & USA & 6038 & 389616 & 2 & 64.53 & 1 \\
\hline $\mathbf{3 .}$ & Korea & 2379 & 59467 & 7 & 52.65 & 5 \\
\hline $\mathbf{4 .}$ & Japan & 2366 & 121829 & 3 & 25.00 & 16 \\
\hline $\mathbf{5 .}$ & Germany & 2307 & 83968 & 4 & 51.49 & 6 \\
\hline $\mathbf{6 .}$ & India & 1938 & 34501 & 12 & 32.85 & 13 \\
\hline $\mathbf{7 .}$ & Australia & 1372 & 72236 & 5 & 36.40 & 11 \\
\hline $\mathbf{8 .}$ & UK & 1265 & 51437 & 8 & 40.66 & 7 \\
\hline $\mathbf{9 .}$ & Singapore & 1036 & 61182 & 6 & 17.80 & 19 \\
\hline $\mathbf{1 0 .}$ & Canada & 999 & 39442 & 11 & 35.78 & 12 \\
\hline
\end{tabular}

\subsection{Subject analysis}

\subsubsection{Keywords of subject}

Based on retrieved literatures, the keywords are analyzed with the tool TDA to exclude invalid concepts and obtain high-frequency keywords related to the hydrogen production with water electrolysis. The keywords of the subject are divided into four types, including reaction principle of the hydrogen production with water electrolysis, catalysis, electrode material, and structure, to conduct filtering, clustering and correlation analysis and obtain the hotspot subject and correlation between subjects, as shown in Table 3. The reaction principles mainly include water separation, hydrogen evolution reaction, oxygen evolution reaction, oxidation-reduction reaction, density functional theory, electrolytic deposition, and other reactions related to the hydrogen production with water electrolysis. The catalysis mainly includes electrocatalysis, electrocatalyst, photocatalysis, photocatalyst, heterogeneous catalysis, photoelectrocatalysis, and other related concepts. The electrode materials mainly include $\mathrm{TiO}, \mathrm{CoO}, \mathrm{CoP}, \mathrm{MoS} 2$, $\mathrm{MoC}, \mathrm{Pt}, \mathrm{Ru}$, Graphene, IrO, perovskite, etc. The structure is mainly related to nanostructure, nano particle, nanosheet, nano-composite, nanowire, 2D material, and coreshell structure, etc.

Table 3 Analysis of frequency terms in the field of hydrogen production with water electrolysis

\begin{tabular}{c|cccc}
\hline $\begin{array}{c}\text { Subject } \\
\text { classification }\end{array}$ & $\begin{array}{c}\text { High-frequency keywords } \\
\text { (English) }\end{array}$ & $\begin{array}{c}\text { Term } \\
\text { frequency }\end{array}$ & High-frequency keywords (English) & $\begin{array}{c}\text { Term } \\
\text { frequency }\end{array}$ \\
\hline Reaction \\
principle & Water splitting & 4703 & Photoelectrochemical & 651 \\
& Hydrogen evolution reaction & 3947 & Oxygen evolution & 644 \\
& Oxygen evolution reaction & 3125 & Electrochemistry & 584 \\
& Hydrogen evolution & 1201 & Photoelectrochemical water splitting & 559 \\
& Hydrogen production & 1147 & Electrodeposition & 536 \\
& Hydrogen & 1070 & water oxidation & 505 \\
& Oxygen reduction reaction & 832 & Overall water splitting & 467 \\
& Water electrolysis & 671 & Density functional theory & 453 \\
\hline Catalysis & Photocatalysis & 2219 & Bifunctional electrocatalyst & 342 \\
& Electrocatalysis & 1938 & Bifunctional catalyst & 188 \\
& Electrocatalyst & 1676 & heterogeneous catalysis & 155 \\
& Catalysis & 497 & Cocatalyst & 141 \\
& Photoanode & 349 & Photoelectrocatalysis & 115 \\
\hline Electrode & Metal-organic frameworks & 469 & Carbon nanotubes & 216 \\
& Graphene & 390 & Thin films & 211 \\
& TiO2 & 344 & Molybdenum disulfide & 200 \\
& MoS2 & 217 & cobalt & 194 \\
& Heterostructure & Perovskite & 182 \\
& Hematite & 259 & Platinum & 172 \\
& Heterojunction & 250 & Reduced graphene oxide & 168 \\
& & & & \\
& & & &
\end{tabular}




\begin{tabular}{c|cccc}
\hline $\begin{array}{c}\text { Subject } \\
\text { classification }\end{array}$ & $\begin{array}{c}\text { High-frequency keywords } \\
\text { (English) }\end{array}$ & $\begin{array}{c}\text { Term } \\
\text { frequency }\end{array}$ & High-frequency keywords (English) & $\begin{array}{c}\text { Term } \\
\text { frequency }\end{array}$ \\
\hline \multirow{3}{*}{ Structure } & Cobalt oxide & 248 & Molybdenum carbide & 153 \\
& nickel & 243 & Titanium dioxide & 153 \\
& nanostructures & 369 & 2D materials & 134 \\
& Nanoparticles & 308 & Nanorods & 113 \\
& Nanosheets & 247 & Nanotubes & 112 \\
& Nanocomposites & 198 & Core-shell structure & 111 \\
& nanowires & 141 & Core-shell & 103 \\
\hline
\end{tabular}

\section{Conclusions}

The hydrogen production with water electrolysis was studied initially from 1990s internationally and few studies were developed before 1990. The annual average number of articles of the field exceeded 1000 after 2013 and increased with growth rate $25 \%$.

Over 130 countries and regions around the world have developed studies related to the hydrogen production with water electrolysis. Various institutions of each country have made wide cooperation, including domestic and international cooperation. China is dominant in the study of this field. Among the top 20 institutions, there are 17 institutions from China. Although China has made a lot of achievements in the field of hydrogen production with water electrolysis, influences of and attentions to the field shall be further strengthened.

The studies related to the hydrogen production with water electrolysis now mainly focus on the reaction, catalytic material, photo-electrochemistry, and structure.

\section{References}

1. Su Xiuli, Liao Wenjun \& Li Yan. The opportunity and challenge of step-by-step hydrogen production with water electrolysis [J]. Energy Storage Science and Technology, 2021,10(01):87-95.

2. Lonis Francesco, Tola Vittorio, Cau Giorgio. Assessment of integrated energy systems for the production and use of renewable methanol by water electrolysis and $\mathrm{CO} 2$ hydrogenation. 2021, 285:119160.

3. Li Tongfei, Luo Gan, Liu Qianyu, et al. Encapsulation of NiCo nanoparticles into foam-like porous N,Pcodoped carbon nanosheets: Electronic and architectural dual regulations toward high-efficiency water electrolysis. 2021, 410(prepublish):128325-.

4. Boppella Ramireddy, Tan Jeiwan, Yun Juwon, et al. Anion-mediated transition metal electrocatalysts for efficient water electrolysis: Recent advances and future perspectives. 2021, 427:213552-. 